\title{
USO DO ÍNDICE GALDIT PARA AVALIAÇÃO DA VULNERABI- LIDADE À SALINIZAÇÃO DO AQUÍFERO BOA VIAGEM - REGIÃO METROPOLITANA DO RECIFE
}

\author{
USE OF GALDIT INDEX TO EVALUATE SALINIZATION VULNERABI- \\ LITY IN BOA VIAGEM AQUIFER - RECIFE METROPOLITAN REGION
}

\author{
Antonio Freire Costa Sobrinho ${ }^{1}$; Jaime Joaquim da Silva Pereira Cabral ${ }^{2}$; Anderson Luiz Ribeiro de \\ Paiva $^{3}$; Suzana Maria Gico Lima Montenegro ${ }^{4}$
}

Artigo recebido em: 13/12/2014 e aceito para publicação em: 06/03/2015.

DOI: http://dx.doi.org/10.14295/ras.v29i1.28016

\begin{abstract}
Recife plain is a estuarine region which has an average elevation ranging from 2 to $10 \mathrm{~m}$, being framed by topographic elevation shaped as small hills. Recife area is crossed by several rivers, streams and wetlands and is a highly sensitive area to tidal movements. Recife has its aquifers bordered by the Atlantic coast, with salinization risk. The aquifer system in Recife Metropolitan Region is composed by the unconfined Boa Viagem aquifer, which overlaps two semi-confined aquifers, the Cabo and the Beberibe, recharge to these two deep aquifer percolates mainly from the unconfined Boa Viagem aquifer. For analysing the degree of vulnerability of this aquifer to sea water intrusion, a model based on indicators to assess and quantify the magnitude of the vulnerability of coastal aquifers to seawater intrusion was used. The GALDIT method of mapping the vulnerability of the aquifer to sea water intrusion has been successfully used to assess the degree of vulnerability of coastal aquifers in many regions around the world. As a result of the application, maps are generated, and can be used as a tool for the management of coastal groundwater resources. Almost all the region of Boa Viagem aquifer has presented risk ranging from moderated to high.
\end{abstract}

Keywords: Coastal Aquifer. Saltwater Intrusion. Shallow Aquifer. GALDIT Index.

Resumo - A planície do Recife consiste numa região estuarina que apresenta cota média de elevação variando de 2 a $10 \mathrm{~m}$, sendo circundada por elevações topográficas em forma de morros de pequenas altitudes. A área apresenta uma ampla malha hidrográfica com vários rios, córregos e mangues, e é uma região altamente sensível aos movimentos de maré e que tem seus aquíferos muito próximos das águas do mar, com elevada possibilidade de salinização. O sistema aquífero da Região Metropolitana do Recife (RMR) é composto por um aquífero livre, o Boa Viagem, que se sobrepõe aos dois aquíferos semiconfinados, o Cabo e o Beberibe, e parte da recarga destes dois aquíferos profundos, vem de percolação pelo aquífero livre Boa Viagem. Para que seja feita uma verificação do grau de vulnerabilidade à salinização desse aquífero, foi utilizado um modelo baseado em indicadores para avaliar e quantificar a magnitude da vulnerabilidade de aquíferos costeiros à intrusão da água do mar. O método GALDIT tem sido usado com sucesso para avaliar o grau de vulnerabilidade de aquíferos costeiros em várias regiões pelo mundo. Como resultado da aplicação, mapas foram gerados para cada indicador do índice GALDIT, e podem ser usados como uma ferramenta para a gestão dos recursos hídricos subterrâneos costeiros. Observouse que a região do aquífero Boa Viagem na RMR possui praticamente toda a área com perigo de salinização de moderado a alto.

Palavras-chaves: Aquífero Costeiro. Intrusão Marinha. Aquífero Freático. Índice GALDIT.

\section{INTRODUÇÃO}

O problema da salinização de poços em Recife vem sendo estudado ao longo de mais de uma década por um conjunto de pesquisadores, entre eles geólogos, engenheiros, químicos buscando um melhor entendimento da questão (COSTA et al., 1998; COSTA et al., 2003; PAI-

\footnotetext{
${ }^{1}$ Universidade Federal de Pernambuco - UFPE, Centro de Tecnologia e Geociências - CTG (costasobrinhoaf@ gmail.com)

${ }^{2}$ Universidade Federal de Pernambuco - UFPE, Centro de Tecnologia e Geociências - CTG (jcabral@ufpe.br)

${ }^{3}$ Universidade Federal de Pernambuco - UFPE, Centro Acadêmico do Agreste - CAA (anderson.paiva@ufpe.br)

${ }^{4}$ Universidade Federal de Pernambuco - UFPE, Centro de Tecnologia e Geociências - CTG (suzanam@ufpe.br)
} 
VA et al., 2003; MONTENEGRO et al., 2008).

A grande heterogeneidade dos tipos litológicos, tanto horizontalmente como verticalmente dificulta um pouco a formatação de um modelo conceitual, de maneira que gradualmente, cada nova informação vai contribuindo para o conhecimento do sistema aquífero como um todo.

A explotação excessiva de aquíferos costeiros na região, em desequilíbrio com o processo de recarga que sofre com avanço de áreas impermeabilizadas, coloca o sistema sob perigo de salinização por diversos processos (COSTA, 2000). Para melhor clareza das informações adiante, é importante analisar a diferença entre vulnerabilidade, risco e perigo, que algumas vezes pode apresentar divergências de acordo com o tema de estudo.

O conceito de vulnerabilidade de águas subterrâneas, segundo Shouyu \& Guangtao (2003), foi introduzido no final dos anos 1960 por Margat, e muitas abordagens diferentes têm sido desenvolvidas para avaliar o problema. Para Chachadi et al. (2003), o termo "vulnerabilidade" foi definido e usado antes da área de recursos hídricos, mas dentro do contexto do sistema de avaliação de desempenho, por exemplo, a definição dada por Hashimoto et al. (1982) em uma análise do desempenho do sistema, que se concentra em caso de falha do sistema, foi apresentada por definir três conceitos que fornecem medidas úteis do desempenho do sistema: (i) Qual a probabilidade de o sistema estar a falhar que é medida por sua confiabilidade; (ii) A rapidez com que o sistema retorna a um estado satisfatório uma vez que ocorreu uma falha que é expressa pela sua resiliência e, (iii) Quão grave as consequências de prováveis falhas podem ser medido pela sua "vulnerabilidade".

Para Chachadi (2005), este conceito de vulnerabilidade definido no contexto do desempenho do sistema pode também ser utilizado no contexto da poluição das águas subterrâneas devido à intrusão de água salgada. Entretanto, é importante deixar claro a distinção entre vulnerabilidade e perigo. Vulnerabilidade é inerente ao aquífero, mas o perigo de salinização é determinado não só pelas características intrínsecas do aquífero, que são relativamente estáticas e pouco mutáveis, mas também sobre a existência de atividades intensivas de bombeamento de águas subterrâneas ou aumento do nível do mar ao longo da costa, que são fatores dinâmicos.

No presente texto, está sendo utilizada a conceituação proposta por Foster et al. (2006): vulnerabilidade refere-se as propriedades intrín117 secas do aquífero que o torna susceptível de sofrer poluição; e perigo refere-se a probabilidade de aquífero sofrer contaminação analisando conjuntamente a vulnerabilidade do aquífero e a presença de poluentes nas proximidades.

Evitou-se usar a palavra risco pelo fato de algumas áreas profissionais calcularem o risco multiplicando o perigo pela escala do impacto. Sendo assim, aqui no Brasil a tendência é que a palavra perigo substitua a palavra risco usada anteriormente pelos hidrogeólogos.

Para o uso sustentável do aquífero é importante avaliar sua vulnerabilidade. Muitas análises de vulnerabilidade de aquíferos têm sido realizadas em diversos países utilizando os métodos denominados de GOD (FOSTER \& HIRATA, 2002) e de DRASTIC (ALLER et al., 1987), que computam um conjunto de indicadores influenciadores na propagação de um poluente após sua deposição no solo.

No entanto, em aquíferos costeiros o perigo de poluição salina pode ocorrer por diversas direções, como por exemplo horizontalmente por percolação vindo do mar diretamente para o aquífero ou verticalmente vindo de camadas superiores salinizadas como manguezais e estuários.

Para aquíferos costeiros, é necessário a utilização de uma metodologia apropriada para mapear a distribuição espacial das áreas litorâneas em relação à vulnerabilidade potencial de intrusão da água do mar, que leve em conta os fatores hidrogeológicos, principalmente aqueles que podem ser obtidos com certa facilidade nos órgãos gestores de recursos hídricos ou através de experimentos simples e rápidos.

O presente estudo foi aplicado ao aquífero livre Boa Viagem que domina praticamente toda região da planície do Recife (capital do estado de Pernambuco), onde se concentra a maior densidade da população da Região Metropolitana do Recife (RMR). Em passado, não muito distante, parte do abastecimento desta população provinha de poços rasos, instalados neste aquífero. Mas, devido principalmente à contaminação pelos poluentes advindos das fossas sépticas, estes poços passaram apenas para utilizações secundárias, tais como regas, lavagem de pátios, etc.

Mesmo não sendo usada para fins mais nobres, a demanda pelas águas do aquífero livre Boa Viagem vem sendo crescente, a cada ano que passa. Tal retirada em excesso, cria condições para que haja salinização, quer seja diretamente do mar ou mesmo indiretamente pelos rios, canais, mangues e região estuarina.

Chachadi et al. (2003) desenvolveram a Águas Subterrâneas (2015) 29(1): 116-128. 
metodologia GALDIT para representar um índice de vulnerabilidade específico para salinização de aquíferos costeiros que pudesse vir a substituir, nestas situações, o Índice DRASTIC (ALLER et al., 1987), criado inicialmente para agricultura e adotado nos EUA, Canadá e África do Sul, e que foi sugerido como um critério padrão em procedimentos de avaliação de vulnerabilidade de águas subterrâneas dos Estados Membros da União Europeia (UE) conforme Lobo-Ferreira \& Cabral (1991); Lobo-Ferreira (1999); Shouyu \& Guangtao (2003).

$\mathrm{Na}$ avaliação do índice DRASTIC, são selecionados sete fatores que regem a vulnerabilidade subterrânea: [Depth to groundwater] (profundidade para o meio aquífero); [ Recharge] (recarga); [Aquifer mídia] (meio aquífero); [Soil mídia] (tipo de solo); [Topography] (topografia); [Impact] (da mídia zona vadosa), e [Conductivity](condutividade hidráulica do aquífero). Cada fator tem um peso, fixo e relativo, prédeterminado, que reflete sua importância relativa para a vulnerabilidade. Os fatores mais importantes têm um peso de 5 (cinco) e os menos significativos têm peso de 1 (um). A vulnerabilidade do aquífero pode ser reconhecida pelos valores padrão de 10 níveis em relação aos 7 fatores.

Para Chachadi et al. (2003), o GALDIT apresenta as vantagens de usar os dados existentes e tem duas partes principais: (i) a designação de unidades mapeáveis, denominado configurações hidrogeológicas, e (ii) a superposição de um sistema de classificação.

Para avaliar as condições de vulnerabilidade do aquífero livre Boa Viagem, foi feita a aplicação da metodologia GALDIT. É oportuno lembrar que o aquífero livre Boa Viagem sobrepõe os aquíferos semiconfinados Cabo e Beberibe - que são muito importantes no abastecimento de água para consumo doméstico da RMR, e muito contribui na recarga destes.

\section{MATERIAIS E MÉTODOS}

\subsection{Descrição da Área do Estudo}

$\mathrm{O}$ foco do estudo foi o aquífero Boa Viagem formado por sedimentos de origem fluvial e marítima. A área de estudo situa-se na Planície do Recife (Figura 1), área mais populosa da Região Metropolitana do Recife (RMR), de acordo com o Instituto Brasileiro de Geografia e Estatística (IBGE, 2010). O aquífero costeiro Boa Viagem comporta-se como aquífero livre e se sobrepõe aos aquíferos semiconfinados Beberibe e Cabo.
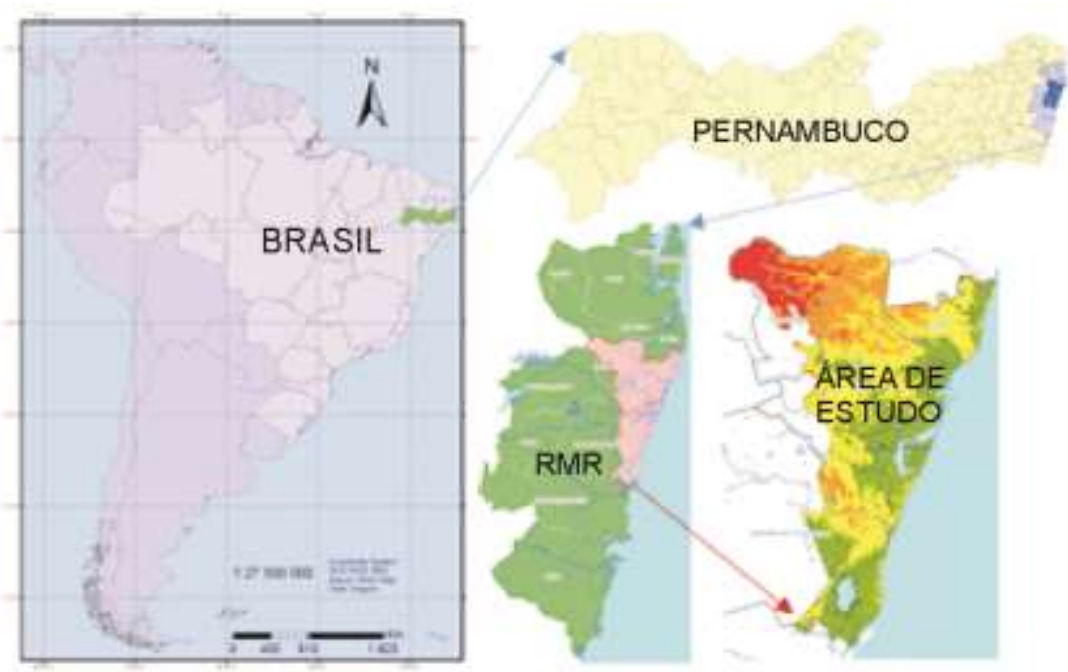

Figura 1 - Localização do Estado do Pernambuco e Região Metropolitana do Recife (RMR) Figure 1 - Location of Pernambuco State and Metropolitan Region of Recife

Segundo Costa Filho (1997), a RMR encontra-se em uma base construída de depósitos de sedimentos quaternários, que coincidem com as planícies de inundação dos vales de antigos rios e riachos. Situada nos limites geográficos de ocorrência sedimentares da bacia do Cabo e da bacia Pernambuco-Paraíba.

O clima da área é quente e úmido, está situada na zona fisiográfica do litoral com precipitação pluviométrica média de $2.450,7 \mathrm{~mm} / \mathrm{ano}$, 
apresentando um período mais chuvoso que vai de março a agosto (sete meses), com valor máximo em julho $(389,7 \mathrm{~mm})$ e um período mais seco, de setembro a fevereiro (cinco meses) com o valor mínimo em novembro $(45,2 \mathrm{~mm})$ e o valor médio mensal de $204,2 \mathrm{~mm}$. Os dados climáticos foram obtidos da Estação Meteorológica do Recife (Curado), com uma série histórica de 30 anos, para o período de 1961 a 1990 (INMET, 2012).

A evaporação potencial anual alcança valores de $1.323,4 \mathrm{~mm}$, superando a precipitação pluviométrica entre os meses de setembro e fevereiro. No entanto, a média anual da pluviometria supera a da evaporação em $1.127,3 \mathrm{~mm}$. A temperatura média anual é de $25,5^{\circ} \mathrm{C}$, com valor máximo médio mensal de $26,6^{\circ} \mathrm{C}$ nos meses de janeiro e fevereiro e média mínima mensal de $23,9^{\circ} \mathrm{C}$ no mês de julho.

A umidade relativa do ar atinge um valor médio mensal de $79,8 \%$. Este alto valor reflete a proximidade da região com o oceano, recebendo as massas de ar úmido, ali geradas e trazidas para a região costeira do continente (FARIAS, 2003).
Com relação à geologia, a planície do Recife está inserida geotectonicamente na bacia sedimentar Pernambuco/Paraíba (norte do Lineamento Pernambuco) e bacia do Cabo (sul do Lineamento Pernambuco). Estas unidades afloram na porção oeste bordejando as duas bacias sedimentares com mergulhos para leste com inclinação de $28 \mathrm{~m} / \mathrm{km}$. Litologicamente o embasamento é constituído de corpos ígneos, sequência parametamórfica e o complexo gnáissicomigmatítico (MOHRIAK, 2003).

A planície do Recife apresenta ao norte do Lineamento Pernambuco a sequência aquífero livre Boa Viagem - aquífero semiconfinado Beberibe, tendo como substrato impermeável o embasamento cristalino; e a sul do Lineamento Pernambuco a sequência aquífero livre Boa Viagem - aquífero semiconfinado Cabo tendo como substrato impermeável o derrame vulcânico da Suíte Ipojuca (COSTA et al., 1998). As tabelas 1 e 2 apresentam algumas características do aquífero Boa Viagem, objeto de estudo.

Tabela 1 - Coluna Litoestratigráfica e parâmetros hidrodinâmicos e geométricos do aquífero livre Boa Viagem - RMR

Table 1 - Litostratigraphic column and hydrodinamics and geometrics parameters of phreatic Boa Viagem aquifer - RMR

\begin{tabular}{|c|c|c|}
\hline \multicolumn{2}{|c|}{ Aquífero $\rightarrow$} & Boa Viagem \\
\hline \multirow{4}{*}{ Coluna Litoestratigráfica } & Idade & Quaternário \\
\hline & Espessura média & $40 \mathrm{~m}$ \\
\hline & Constituição Litológica & Areias, siltes e argilas \\
\hline & Transmissividade & $7,0 \times 10^{-3} \mathrm{~m}^{2} / \mathrm{s}$ \\
\hline \multirow{3}{*}{ Parâmetros Hidrodinâmicos } & Condutividade Hidráulica & $1,7 \times 10^{-4} \mathrm{~m} / \mathrm{s}$ \\
\hline & Porosidade eficaz & $1,0 \times 10^{-1}$ \\
\hline & Coef. de armazenamento & - \\
\hline \multirow{2}{*}{ Parâmetros Geométricos } & Área & $112 \times 10^{6} \mathrm{~m}^{2}$ \\
\hline & Espessura saturada & $40 \mathrm{~m}$ \\
\hline
\end{tabular}

Fonte: Costa et al. (2011)

Tabela 2 - Estimativa do balanço anual de entradas e saídas do aquífero Boa Viagem - RMR

Table 2 - Estimate of in and out annual balance of Boa Viagem aquifer - RMR

\begin{tabular}{|c|c|c|}
\hline Aquífero $\rightarrow$ & & Boa Viagem \\
\hline \multirow{4}{*}{$\begin{array}{l}\text { Balanço Anual de } \\
\text { Entradas x Saídas }\end{array}$} & (a) Recarga & $45,76 \times 10^{6} \mathrm{~m}^{3} /$ ano \\
\hline & (b) Exutório natural & $11,64 \times 10^{6} \mathrm{~m}^{3} /$ ano \\
\hline & (c) Bombeamento dos poços & $21,63 \times 10^{6} \mathrm{~m}^{3} /$ ano \\
\hline & (d) Balanço anual $(=\mathrm{a}-(\mathrm{b}+\mathrm{c}))$ & $12,49 \times 10^{6} \mathrm{~m}^{3} /$ ano \\
\hline
\end{tabular}

Fonte: Costa et al. (2011) 
Em Recife já existem diversos poços salinizados. Muitos destes devido a erros de construção, quando não havia fiscalização adequada, e desta maneira o espaço anelar do poço não era devidamente isolado por cimentação e, o que ocorria era a construção de furos (assim caracterizados por não obedecer às técnicas mínimas de construção de poços), que podem proporcionar a verticalização rápida de fluxo, que poderão levar a alterações por processos de mistura entre as águas das diversas formações perpassadas pelos mesmos (CABRAL \& MONTENEGRO, 2004). Nos bairros centrais de Recife, uma causa provável é a infiltração de águas do rio Capibaribe que no trecho final do baixo curso apresenta suas águas misturadas com a água do mar (Figura 2). O bombeamento excessivo dos poços próximos ao rio induz uma recarga do aquífero com águas salinizadas do rio (CABRAL et al., 2004).

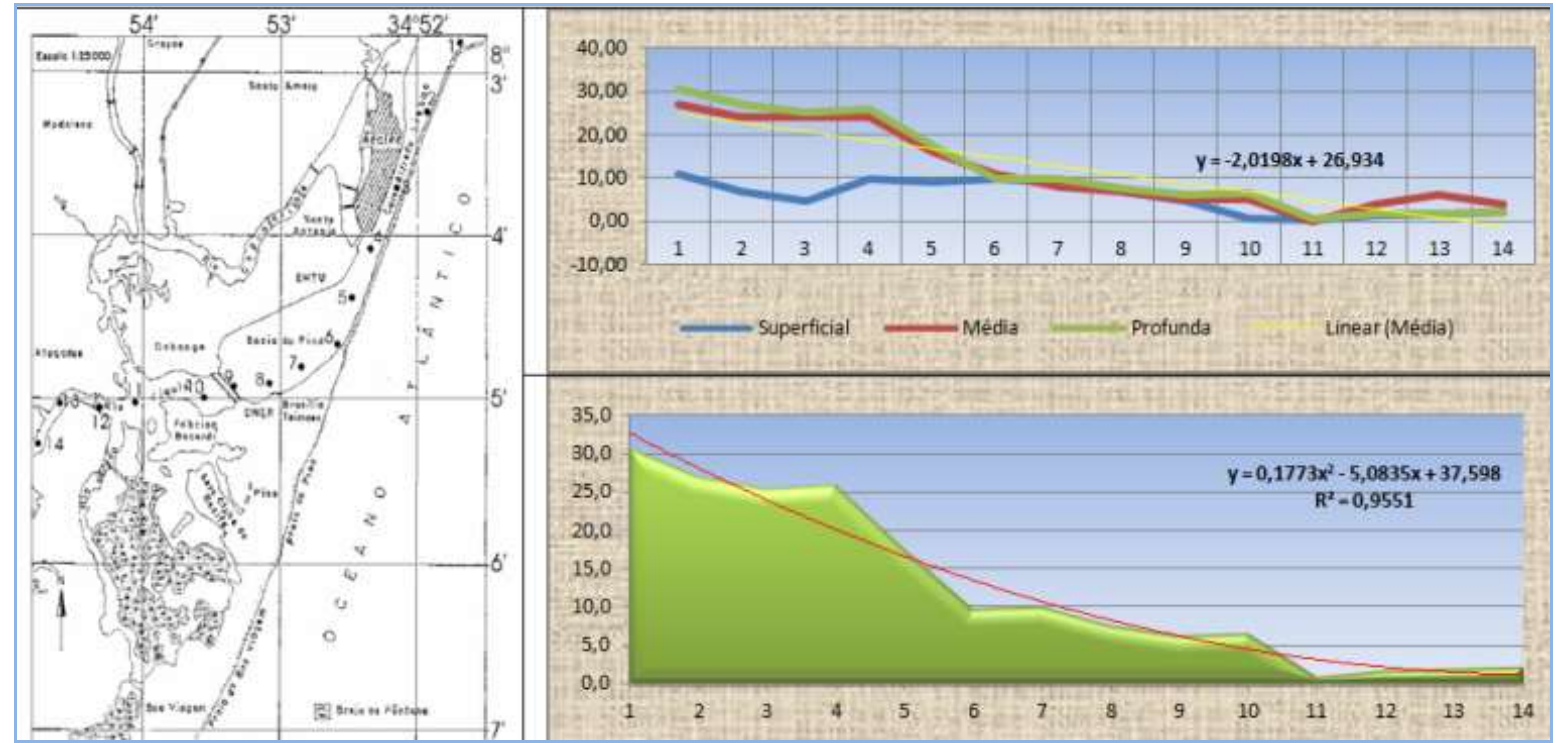

Figura 2 - Rio Capibaribe: estações e gráficos de salinização

Figure 2 - Capibaribe river: stations and salinization graphics

Fonte: Nascimento et al. (2003)

*Eixo horizontal são as estações indicadas no mapa à esquerda, eixo vertical salinidade

Em particular, a Planície do Recife apresenta também salinização dos aquíferos, onde se verifica área com superexplotação (CABRAL et al., 2008). A ausência de camadas confinantes, em alguns pontos dessa área, deixa o aquífero vulnerável à contaminação de superfície, principalmente onde ocorre intenso bombeamento de poços (OLIVEIRA, 1994).

\subsection{Metodologia GALDIT}

A metodologia GALDIT utiliza seis parâmetros, considerados necessários para desenvolver um sistema de mapeamento que, segundo os seus criadores (CHACHADI \& LOBOFERREIRA, 2001), "é simples o suficiente para aplicar usando os dados disponíveis, e ainda assim capaz de fazer melhor uso dos dados disponíveis de uma maneira tecnicamente válida e útil". A adoção de um índice tem a vantagem de, em princípio, eliminar ou minimizar a subjetividade no processo de classificação (CHACHADI, 2005).
As características físicas são inerentes a cada configuração hidrogeológica e, afetam o potencial de intrusão de água salgada. Chachadi et al. (2003) levantaram os fatores de controle mais importantes, mapeáveis, na intrusão de água do mar que foram encontrados para serem os seguintes: água subterrânea (Groundwater), meio aquífero (Aquifer), nível freático (Level), distância à costa (Distance), status de intrusão de água salina existente (Impact), espessura do aquífero (Thickness), que são parâmetros mensuráveis.

Todos os dados são manipulados com a utilização de planilha eletrônica e uma ferramenta de SIG (sistema de informações georreferenciado), que são utilizados para confeccionar os mapas temáticos nos quais são dispostos os valores para cada índice a área onde este se encontra aplicado.

Para verificar os valores finais do índice de vulnerabilidade GALDIT é necessário fazer os cálculos de uma relação ponderada (equação 1), onde são considerados pesos para cada índice: 
$\mathrm{G}=1, \mathrm{~A}=3, \mathrm{~L}=4, \mathrm{D}=4, \mathrm{I}=1$ e $\mathrm{T}=2$. Na Tabela 3 é possível visualizar todos os fatores com respecti-

Índice GALDIT $=\sum_{i=1}^{6}\left\{\left(W_{i}\right) \cdot R_{i}\right\} / \sum_{i=1}^{6} W_{i}$

Tabela 3 - Fatores para os indicadores da metodologia GALDIT.

Tabela 3 - Factors to indicators of GALDIT methodology

\section{Fator}

Peso

Variáveis indicadoras

Classe

G águas subterrâneas) A
Aquifer (condutividade hidráulica do
aquífero) (m/dia)

\section{$\mathbf{L}$}

Height of ground water Level above msl (Altura do nível do lençol freático acima do nível do mar) (m)

\section{D}

Distance of the point in question from shore / High tide (Distância do ponto em questão à costa / Maré alta) (m)

I

Impact status of existing seawater intrusion [Based on Range of $\mathrm{Cl} /\left(\mathrm{HCO}_{3}+\mathrm{CO}_{3}\right)$ ratio in epm in groundwater] (estado do Impacto existente da intrusão de água do mar)

\section{$\mathbf{T}$}

Aquifer Thickness (saturated) [espessura do aquífero (saturado)] (m) vos pesos e classes de importância.

\begin{tabular}{|c|c|c|}
\hline \multirow{4}{*}{1} & Aquífero Confinado & $\mathbf{1 0 , 0}$ \\
\hline & Aquífero não Confinado & 7,5 \\
\hline & Aquífero semiconfinado & 5,0 \\
\hline & $\begin{array}{l}\text { Aquífero Limitado (recarga e/ou limite im- } \\
\text { permeável alinhado paralelamente à costa) }\end{array}$ & 2,5 \\
\hline \multirow{4}{*}{3} & Alta $(>40)$ & 10,0 \\
\hline & Média (10-40) & 7,5 \\
\hline & Baixa $(5-10)$ & 5,0 \\
\hline & Muito Baixa $(<5)$ & 2,5 \\
\hline \multirow{4}{*}{4} & Alto $(<1,0)$ & 10,0 \\
\hline & Médio $(1,0-1,5)$ & 7,5 \\
\hline & Baixo $(1,5-2,0)$ & 5,0 \\
\hline & Muito Baixo $(>2,0)$ & 2,5 \\
\hline \multirow{4}{*}{4} & Muito Pequena $(<500)$ & 10,0 \\
\hline & Pequena (500-750) & 7,5 \\
\hline & Média (750-1.000) & 5,0 \\
\hline & Longe $(>1.000)$ & 2,5 \\
\hline \multirow{4}{*}{1} & Alto $(>2,0)$ & 10,0 \\
\hline & Médio $(1,5-2,0)$ & 7,5 \\
\hline & Baixo $(1,0-1,5)$ & 5,0 \\
\hline & Muito Baixo $(<1,0)$ & 2,5 \\
\hline \multirow{4}{*}{2} & Grande $(>10,0)$ & $\mathbf{1 0 , 0}$ \\
\hline & Média $(7,5-10,0)$ & 7,5 \\
\hline & Pequena $(5,0-7,5)$ & 5,0 \\
\hline & Muito Pequena $(<5,0)$ & 2,5 \\
\hline
\end{tabular}

O método concebido por Chachadi \& Lobo-Ferreira (2001) contém três partes significativas: pesos, faixas e classificações de importância. Cada fator GALDIT foi avaliado com relação ao outro para determinar a importância relativa de cada fator. A suposição básica feita no desenvolvimento da ferramenta inclui: que o fundo do aquífero(s) está abaixo do nível médio do mar.

As configurações hidrogeológicas incorporam os principais fatores hidrogeológicos. No caso de Recife, as Tabelas 1 e 2 apresentam os valores que são usados para inferir o potencial de contaminantes para entrar na água do solo. 


\section{RESULTADOS DO MÉTODO GALDIT}

Os vários parâmetros indicadores adotados na evolução da presente ferramenta incluem: identificação de todos os indicadores que influenciam o episódio de invasão de água salinizada. Esta tarefa foi conseguida através de amplas discussões e consultas com especialistas. $\mathrm{O}$ peso indicador: pesos dos indicadores retratam a importância relativa do indicador para o processo de intrusão salina. A seguir são apresentados os resultados para cada parâmetro e ao fim o índice GALDIT gerado.

\section{Parâmetro G: Ocorrência de águas subterrâ- neas (tipo de aquífero)}

O Sistema Aquífero Boa Viagem é um aquífero livre e, como tal, tem classificação GALDIT para o parâmetro G uma atribuição de valor 7,5 (Figura 3).

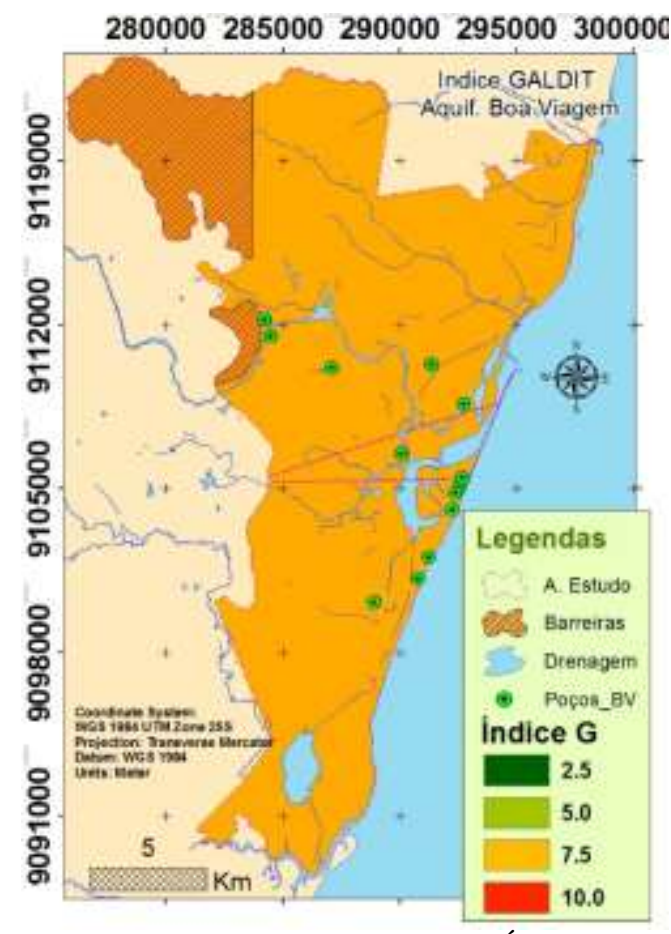

Figura 3 - Mapa do Resultado do Índice G

Figure 3 - Map of G Index result

\section{Parâmetro A: Condutividade Hidráulica do Aquífero}

A condutividade hidráulica do Sistema Aquífero Boa Viagem tem seu valor de 1,7 x 10 $\mathrm{m} / \mathrm{s}$, conforme Costa et al. (2011). Uma vez que não se dispunha de valores conhecidos, para se- rem interpolados para a área do aquífero através de um método de krigagem, que trariam maior precisão nos resultados, este foi atribuído e utilizado como dados de entrada para computação do índice A (Figura 4). 


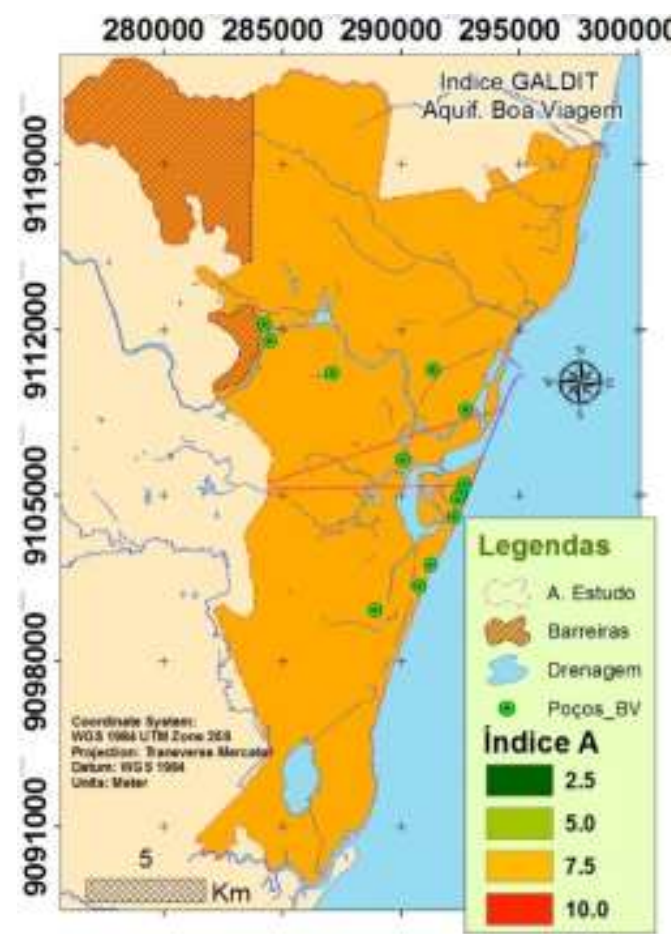

Figura 4 - Mapa do Resultado do Índice A

Figure 4 - Map of A Index result

Parâmetro L: Altura do nível do lençol freático acima do nível do mar

Os valores adotados para este parâmetro foram obtidos através da interpolação para a área do aquífero através de um método de krigagem que interpola uma superfície "raster" a partir de pontos utilizando uma técnica de distância inver- sa ponderada (Inverse Distance Weighted IDW), de valores obtidos de 1.314 poços, bem distribuídos na área de estudo, através do site da CPRM (2012). Assim com os dados da altura do nível do lençol freático acima do nível do mar foi produzido o mapa relativo ao Índice L (Figura 5).

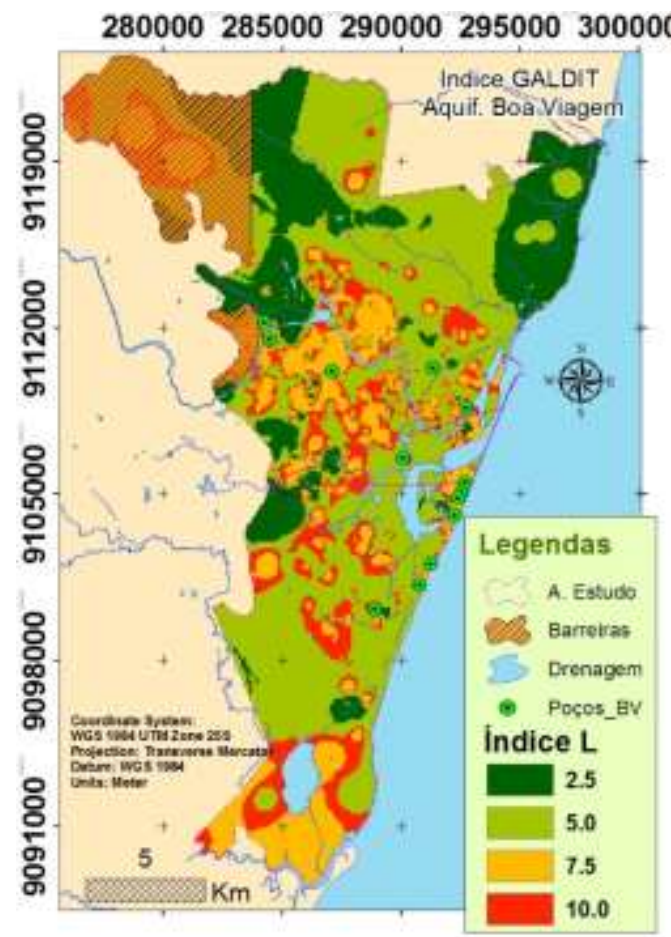

Figura 5 - Mapa do Resultado do Índice L

Figure 5 - Map of L Index result 


\section{Parâmetro D: Distância à costa (ou à corpo d'água salinizado)}

Para este parâmetro o sistema GALDIT determina que seja feito o cálculo de três distâncias perpendiculares à linha da costa que são: 500, 750 e $1.000 \mathrm{~m}$, a partir da linha costeira do aquífero e das margens dos rios em seus trechos salinizados. Como mencionado, as águas de superfície das saídas dos rios na RMR são salobras, a vulnerabilidade a intrusão de água para essas áreas tem os mesmos efeitos práticos negativos que os da zona costeira. A distribuição do parâmetro D é apresentada na Figura 6.

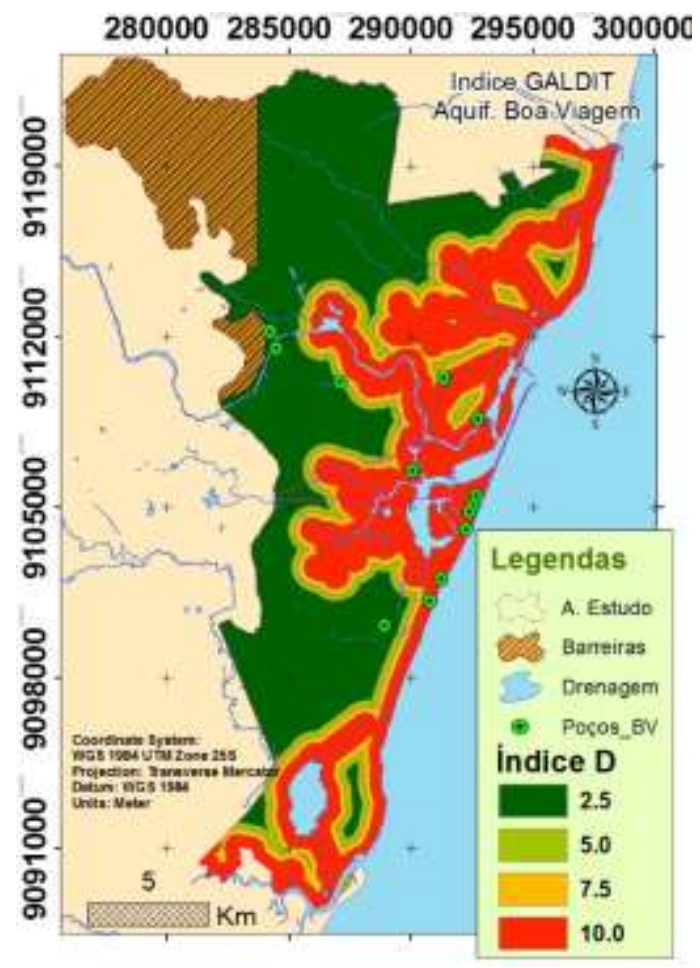

Figura 6 - Mapa do Resultado do Índice D

Figure 6 - Map of D Index result

Parâmetro I: Impacto do estado existente de intrusão salina

Para avaliar este parâmetro a proporção de $\mathrm{Cl} / \mathrm{HCO}_{3}$ foi utilizada com dados de outro projeto do mesmo grupo de pesquisa, na campanha de amostragem em setembro de 2012, que entre outros dados determinou as concentrações desses dois ânions para os 65 poços selecionados, que através da interpolação para a área do aquífero usando um método de krigagem. A distribuição do índice I é apresentada na Figura 7. 


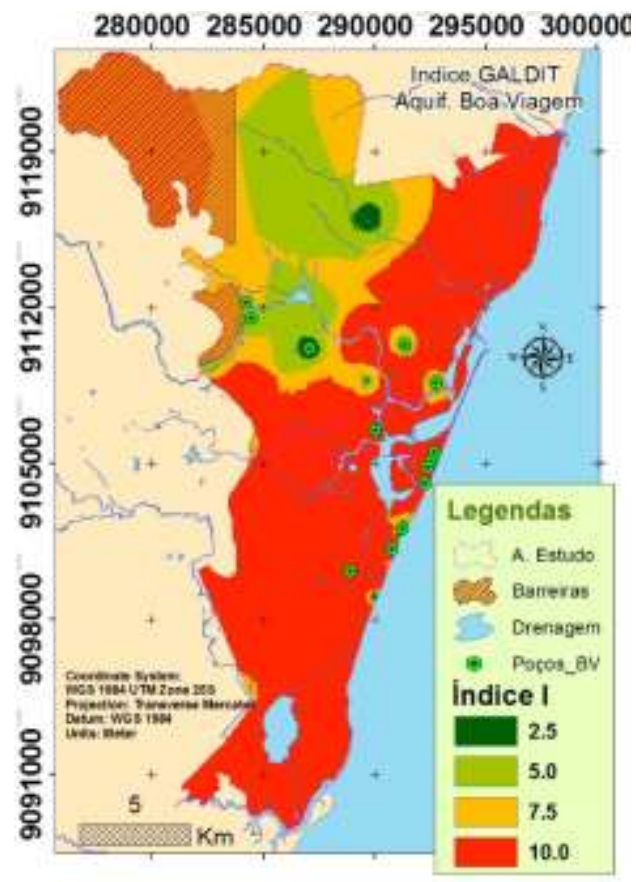

Figura 7 - Mapa do Resultado do Índice I

Figure 7 - Map of I Index result

\section{Parâmetro T: Espessura do Aquífero}

O Sistema Aquífero Boa Viagem tem uma espessura média de $40 \mathrm{~m}$, conforme Costa et al. (2011). Entretanto, com a utilização dos dados de poços da região obtidos no trabalho de Mon- teiro (2000), pode-se realizar a krigagem das cotas de topo e base do aquífero. Dessas informações extraiu-se a espessura que foi posto no mapa do índice $\mathrm{T}$ (Figura 8).

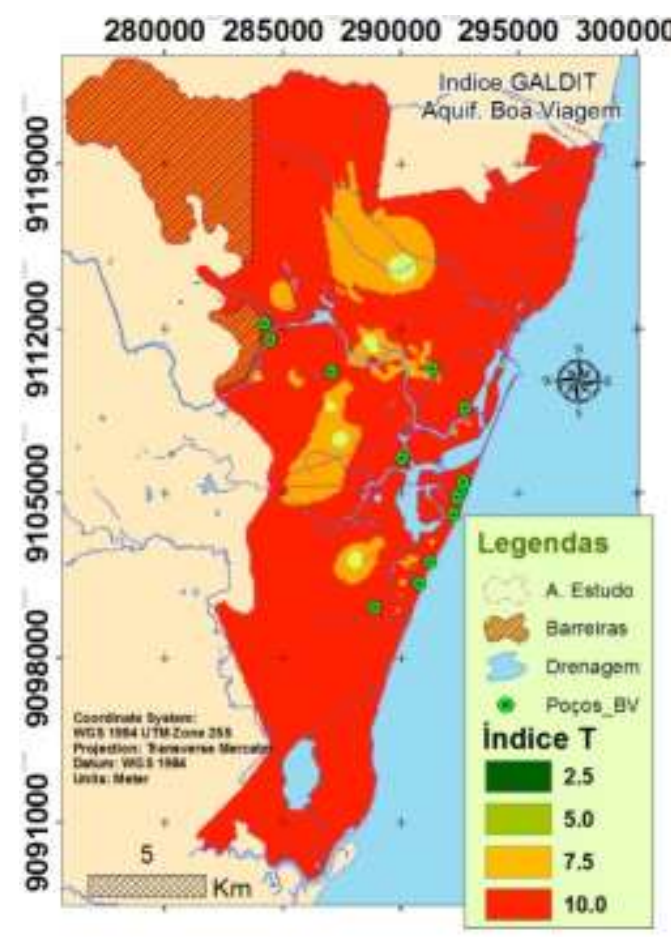

Figura 8 - Mapa do Resultado do Índice T Figure 8 - Map of T Index result 


\section{Resultado e Discussão do Índice GALDIT: categorias de vulnerabilidade}

Com cada um dos Índices GALDIT mapeados em arquivo raster em um aplicativo GIS, foi possível classificar as áreas costeiras em várias categorias de vulnerabilidade à intrusão da água do mar. $\mathrm{O}$ intervalo de pontuações para indexação GALDIT está dividida em três grupos (Tabela 4). Todos os seis indicadores têm quatro classes: $2.5,5,7.5$ e 10 , conforme suas classificações de importância. A Tabela 3 apresenta os valores para classificação detalhada.

Tabela 4 - Faixas de Índice GALDIT

Table 4 - Rangers of GALDIT Index

\begin{tabular}{cc}
\hline Faixas de Índice GALDIT & Classes de Vulnerabilidade \\
\hline$\geq 7,5$ & Alta Vulnerabilidade \\
5,0 a 7,5 & Média Vulnerabilidade \\
$<5,0$ & Baixa Vulnerabilidade \\
\hline
\end{tabular}

Fonte: Chachadi (2005)

O GALDIT leva em conta não só o risco de um poluente vindo da parte superior (como outros métodos tradicionais), mas considera a contribuição lateral vindo do mar ou de corpos d'água superficiais salinizados. No caso de Recife, além do mar que ocupa toda a fronteira leste, temos os estuários de diversos rios, a lagoa Olho d'Água e o parque dos manguezais.

O resultado final pode ser observado na Figura 9, onde quase toda área estudada possui índice GALDIT de moderado a alto. Vindo de norte para sul observa-se o estuário do rio Parati- be (compartilhado com o rio Doce), na parte central os rios Beberibe, Capibaribe e Tejipió, e na parte sul o rio Jaboatão. Como a parte final destes rios atravessa uma região muito plana, a salinidade do mar se propaga até cerca de $8 \mathrm{Km}$ rio a dentro (PAIVA, 2004). Na parte central, observase a faixa azul alargada que corresponde as águas salinizadas da bacia do Pina e do parque dos manguezais. E ao sul, observa-se a lagoa Olho d'Água que tem conexão direta com o mar. Por isso, então, a região de perigo alto prolonga-se bastante para dentro do continente.

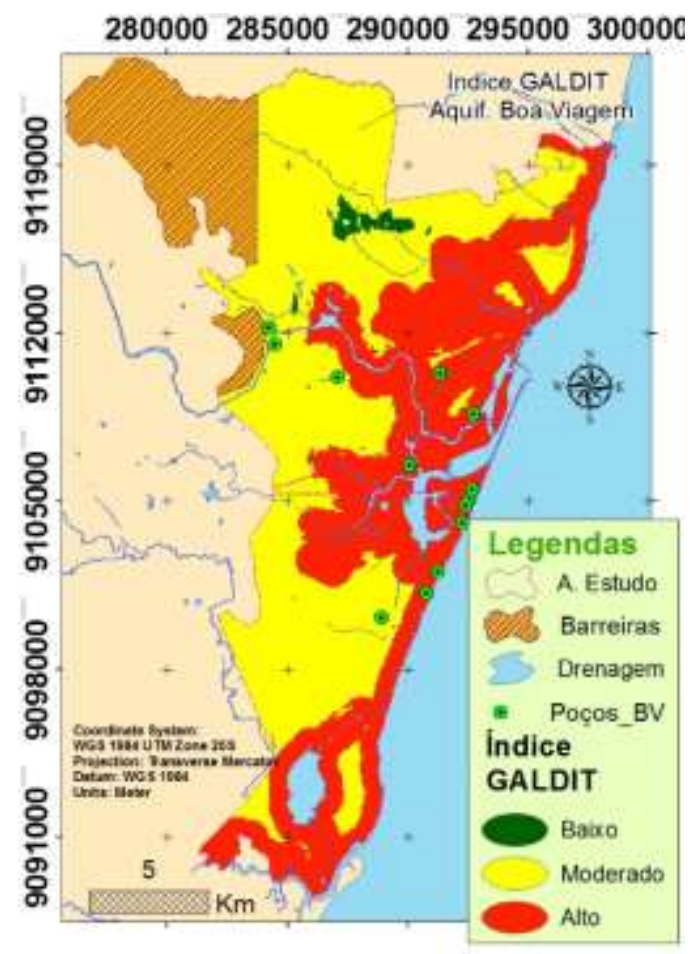

Figura 9 - Mapa do índice GALDIT

Figure 9 - Map of GALDIT Index 


\section{CONSIDERAÇÕES FINAIS}

Para aquíferos costeiros, em que o principal perigo é a contaminação salina, a utilização da metodologia GALDIT para a análise da vulnerabilidade do aquífero é mais indicada do que outras metodologias tradicionais como o GOD ou DRASTIC, voltadas para outros tipos de contaminantes.

Na formulação do método GALDIT os dois fatores de maior influência são a distância em relação a um corpo d'água salinizado (parâmetro D) e a altura da superfície potenciométrica em relação ao nível do mar (parâmetro L). E o terceiro fator mais importante é a condutividade hidráulica (parâmetro A). Na presente aplicação observou que o parâmetro $\mathrm{D}$ foi o de maior relevância.

Após a aplicação da Metodologia GALDIT, vê-se que as áreas mais propensas à salinização, nem sempre são apenas aquelas que se avizinham à costa. Por vezes a salinidade pode ser conduzida pelas calhas dos rios e córregos que por influência dos movimentos de maré conduzem as águas salinas por alguns quilômetros para o interior do continente. E por meio dos mangues estuarinos, as águas salinas podem alcançar os aquíferos freáticos, e por percolação, pode também atingir os aquíferos semiconfinados que se encontram abaixo destes.

\section{REFERÊNCIAS}

ALLER, L.; LEHR, J.H.; PETTY, R. (1987). DRASTIC: A Standardized system of evaluating groundwater pollution potential using hidrogeologic settings. United States:EPA

CABRAL， J.J.S.P.; MONTENEGRO, S.M.G.L. (2004). Salinização em aquíferos costeiros - Estudo de caso na Planície do Recife. In: CABRAL, J.J.S.P.; LOBO FERREIRA, J.P.; MONTENEGRO, S.M.G.L.; COSTA, W.D. (organizadores) Água Subterrânea, aquíferos Costeiros e Aluviões, Vulnerabilidade e Aproveitamento, Tópicos Especiais em Recursos Hídricos, v. 4, Editora UFPE, Recife.

CABRAL， J.J.S.P.; MONTENEGRO, S.M.G.L.; PAIVA, A.L.R.; FARIAS, V.P. (2004). Groundwater salinization in the Central Region of Recife (Brazil) due to brackish water in Capibaribe river at high tide. In: L. Araguás, E. Custódio and M. Manzano (editors). Groundwater and Saline Intrusion. Cartagena, Spain. p.717-727.

CABRAL, J.J.S.P.; FARIAS, V.P.; SOBRAL, M.C.M.; PAIVA, A.L.R.; SANTOS, R.B. (2008).
Além disso, com o avanço horizontal do mar, principalmente na preamar, é possível que (i) algumas áreas de recarga e (ii) até mesmo de localização de poços construídos sejam atingidas. Estas são grandes preocupações, principalmente a segunda, pois levaria a uma rápida contaminação vertical, nos casos de poços abandonados.

A planície do Recife apresenta grandes trechos de mangue e de estuários de rios. Tanto os mangues como os estuários apresentam salinidade elevada, principalmente nos períodos de estiagem, durante a preamar, o que aumenta o perigo de salinização chegar aos aquíferos.

O aquífero Boa Viagem uma vez salinizado também pode exercer influência na contaminação do aquífero sotoposto, aquífero Cabo, importante elemento no abastecimento de água da RMR.

\section{AGRADECIMENTOS}

Os autores agradecem ao CNPQ e FACEPE pelo apoio à linha de pesquisa de salinização de aquíferos e pelas bolsas de pós-graduação e produtividade.

$\mathrm{O}$ primeiro autor agradece à CAPES pela bolsa de doutorado.

Groundwater Management in Recife, DOI: 10.1080/ 02508060801927648, IWRA, Water International, v.33, n.1, p.86-89.

CHACHADI, A.G.; LOBO-FERREIRA, J.P.C. (2001). Sea water intrusion vulnerability mapping of aquifers using GALDIT method, p.143-156 Proceedings of the Workshop on Modelling in Hydrogeology, Anna University, Chennai.

CHACHADI, A.G.; LOBO-FERREIRA, J.P.C.; CHOUDRI, B.S. (2003). Intrusion in Coastal Aquifers Using GALDIT Model. APRH/CEAS, Seminário Sobre Águas Subterrâneas, Lisboa, Fev. 2003.

CHACHADI, A. G. (2005). Seawater Intrusion Mapping Using Modified Galdit Indicator Model - Case Study In Goa. Jalvigyan Sameeksha, v. 20, 2005. Department of Earth Science, Goa University, Goa.

CPRM - Serviço Geológico do Brasil. (2012). SIAGAS - Sistema de Informações de Águas Subterrâneas. ttp://siagasweb.cprm.gov.br/layout/index.php, acessado em dezembro/2012. 
COSTA, W.D. (2000). Riscos potenciais e reais de correntes da superexplotação de águas subterrâneas no Recife - PE. XI Congresso Brasileiro de Águas Subterrâneas. Fortaleza, CE.

COSTA, W.D.; COSTA, H.F.; FERREIRA, C.A.; MORAIS, J.F.S.de, VILLA VERDE, E.R.; COSTA, L.B. (2003). Estudo Hidrogeológico de RecifeOlinda- Camaragibe- Jaboatão dos Guararapes- HIDROREC II. SRH- PE. Recife, 150p.

COSTA, W.D.; MANOEL FILHO, J.; SANTOS, A.C.; COSTA FILHO, W.D.; MONTEIRO, A.B.; SOUZA, F.J.A.de; LOPES, A.V.G.; SANTOS, A.J.C.; SILVA FILHO, M.C.; SILVA, M.J.da (1998). Estudo Hidrogeológico da Região Metropolitana do Recife - Relatório Técnico. v.1, 116p. Convênio IDRCUFPE-FADE.

COSTA W.D.; MONTEIRO A.B.; COSTA FILHO W.D.; SANTOS, A.C. (2011). Condicionamento Hidrogeológico da Explotação do Aquífero Costeiro Boa Viagem. 1st Joint World Congress on Groundwater. ABAS.

COSTA FILHO, W.D. (1997). Estudo Hidroquímico nos aqüíferos da Planície do Recife. Dissertação de Mestrado. Recife-PE, UFPE. 225p.

FARIAS, V.P.de (2003). Estudo das águas subterrâneas na área do Complexo Médico-Empresarial da Ilha do Leite e regiões adjacentes no município de Recife-PE. 78 folhas: Dissertação (mestrado) Universidade Federal de Pernambuco. CFCH. PROPESQ. Gestão e Políticas Ambientais.

FOSTER, S.S.D.; HIRATA, R.C.A. (1988). Groundwater pollution risk evaluation: the methodology using available data. Lima: CEPIS/PAHO/WHO.

IBGE - Instituto Brasileiro de Geografia e Estatística (2010). Censo Demográfico - em www.ibge.org.br acessado em março 2011.

INMET - Instituto Nacional de Meteorologia (2012). $3^{\circ}$ DISTRITO DE METEOROLOGIA (DISME). Boletim com a Normal Climatológica no período 1961 a 1990 em www.inmet.gov.br.

HASHIMOTO, T.; STEDINGER, J.R.; LOUCKS, D.P. (1982). Reliability, Resiliency, and Vulnerability Criteria for Water Resource System Performance Evaluation, Water Resources Research, v.18, n.1, p.14-20.

LOBO-FERREIRA, J.P.; CABRAL, M. (1991) - Proposal for an operational definition of vulnerability of the European community's Atlas of groundwa- ter resources." Meeting of the European Institute for Water, Groundwater Work Group, Brussels, Belgium.

LOBO-FERREIRA, J.P. (1999). The European Union experience on groundwater vulnerability assessment and mapping. COASTIN - A Coastal Policy Research Newsletter n.1, September 1999. New Delhi, TERI, pp8-10.

MOHRIAK, W.U. (2003). Bacias Sedimentares da Margem Continental Brasileira. Sedimentary Basins of the Brazilian Continental Margin. Cap. III Petrobras, Parte I - Geologia. Geologia, Tectônica e Recursos Minerais do Brasil, 87-94. L. A. Bizzi, C. Schobbenhaus, R. M. Vidotti e J. H. Gonçalves (eds.), CPRM, Brasília.

MONTEIRO, A.B. (2000). Modelagem do Fluxo Subterrâneo nos aquíferos da Planície do Recife e seus Encaixes. Dissertação de Mestrado, PósGraduação em Geociências do Centro de Tecnologia e Geociências da Universidade Federal de Pernambuco.

MONTENEGRO, S.M.G.L.; CABRAL， J.J.S.P.; PAIVA, A.L.R.de. (2008). Modelagem da Salinização dos aquíferos da Planície de Recife com Modelo SEAWAT. In: X Simpósio de Recursos Hídricos do Nordeste, 2008, Salvador. Anais do IX SIMPÓSIO DE RECURSOS HÍDRICOS DO NORDESTE.

NASCIMENTO, F.C.R. do; MUNI, K; FEITOSA, F.A.N; ARAÚJO, J.P.de; SILVA, R.M.S.da; SILVA, G.S.da; MONTES, M.J.F. (2003). Disponibilidade nutricional da bacia do Pina e rio Tejipió (Recife-PEBrasil) em relação aos nutrientes e biomassa primária. Tropical Oceanography, v.31, n.2, p149-169.

OLIVEIRA, R.G.de (1994) - Sistema de Informações para Gestão Territorial da Região Metropolitana do Recife - Projeto SINGRE; Levantamento Gravimétrico da Área Sedimentar da Região Metropolitana do Recife. Recife: CPRM/FIDEM

PAIVA, A.L.R.de (2004) - Modelagem Computacional e Análise da Salinização dos aquíferos na Área Central de Recife. UFPE. Recife, Pernambuco. 175p. Dissertação de Mestrado.

PAIVA, A.L.R.de; CABRAL, J.J.S.P.; FARIAS, V.P.; MONTENEGRO, S.M.G.L. (2003) - Problemas de salinização nos aqüíferos costeiros da região central do Recife. Anais do II Congresso sobre Planejamento e Gestão da Zona Costeira dos Países de Expressão Portuguesa. Recife, PE.

SHOUYU, C; GUANGTAO F. (2003). A DRASTICbased fuzzy pattern recognition methodology for groundwater vulnerability evaluation. Hydrological Sciences Journal, v.48, n.2, p.211-220. 